\title{
Exploring the Role of Breast Density on Cancer Prognosis among Women Attending Population-Based Screening Programmes
}

\author{
Laia Domingo, ${ }^{1,2}$ Maria Sala (D), ${ }^{1,2}$ Javier Louro, ${ }^{1,2}$ Marisa Baré,,3,4 ${ }^{2,2}$ esa Barata, ${ }^{5}$ \\ Joana Ferrer, ${ }^{6}$ Maria Carmen Carmona-Garcia, ${ }^{7,8,9}$ Mercè Comas, ${ }^{1,2}$ Xavier Castells, ${ }^{1,2,3}$ \\ and CAMISS Study Group ${ }^{10}$ \\ ${ }^{1}$ Department of Epidemiology and Evaluation, IMIM (Hospital del Mar Medical Research Institute), Passeig Marítim, 25-29, \\ 08003 Barcelona, Spain \\ ${ }^{2}$ Research Network on Health Services in Chronic Diseases (REDISSEC), Barcelona, Spain \\ ${ }^{3}$ Department of Paediatrics, Obstetrics and Gynaecology, Preventive Medicine and Public Health, \\ Universitat Autònoma de Barcelona (UAB), 08193 Bellaterra, Barcelona, Spain \\ ${ }^{4}$ Cancer Screening and Clinical Epidemiology, Corporació Sanitària Parc Taulí, 08208 Sabadell, Spain \\ ${ }^{5}$ General Directorate of Health Care Programmes, Canary Islands Health Service, C/Juan XXIII, 13 35005, \\ Las Palmas de Gran Canaria, Spain \\ ${ }^{6}$ Department of Radiology, Hospital de Santa Caterina, C/Dr. Castany, s/n, 17190 Salt, Girona, Spain \\ ${ }^{7}$ Epidemiology Unit and Girona Cancer Registry, Oncology Coordination Plan, Department of Health, \\ Catalan Institute of Oncology, C/Sol, 15, Barcelona 17004, Girona, Spain \\ ${ }^{8}$ Girona Biomedical Research Institute (IDIBGI), C/Dr Castany s/n, 17190 Salt, Girona, Spain \\ ${ }^{9}$ Department of Medical Oncology, Catalan Institute of Oncology, University Hospital Dr. Josep Trueta, Av França s/n, 17007, \\ Barcelona, Girona, Spain \\ ${ }^{10}$ IMIM (Hospital del Mar Medical Research Institute), Passeig Maritim, 25-29, 08003 Barcelona, Spain
}

Correspondence should be addressed to Maria Sala; 92601@parcdesalutmar.cat

Received 26 April 2019; Accepted 5 August 2019; Published 27 November 2019

Guest Editor: Chia-Jung Li

Copyright (C) 2019 Laia Domingo et al. This is an open access article distributed under the Creative Commons Attribution License, which permits unrestricted use, distribution, and reproduction in any medium, provided the original work is properly cited.

Background. Our aim was to assess the role of breast density on breast cancer mortality and recurrences, considering patient and tumour characteristics and the treatments received among women attending population-based screening programmes. Methods. We conducted a retrospective cohort study among women aged 50-69 years attending population-based screening programmes, diagnosed with invasive breast cancer between 2000 and 2009, and followed up to 2014. Breast density was categorised as low density ( $\leq 25 \%$ dense tissue), intermediate density (25-50\%), and high density ( $\geq 50 \%)$. Cox proportional hazards regression models were fitted to estimate the adjusted hazard ratios (aHR) and $95 \%$ confidence intervals (95\% CI) for death and recurrences, adjusting by patient characteristics, mode of detection (screen-detected vs. interval cancer), and tumour features. Results. The percentage of deaths and recurrences was higher among women with intermediate- and high-density breasts than among women with low-density breasts ( $p=0.011$ for death; $p=0.037$ for recurrences). Adjusted Cox proportional hazards regression models revealed that women with intermediate- and high-density breasts had a higher risk of death than women with low-density breasts, being statistically significant for intermediate densities ( $\mathrm{aHR}=2.19$ [95\% CI: $1.16-4.13]$, aHR $=1.44$ [95\% CI: 0.67-3.1], respectively). No association was found between breast density and recurrences. Conclusions. Breast density was associated with a higher risk of death, but not of recurrences, among women participating in breast cancer screening. These findings reinforce the need to improve screening sensitivity among women with dense breasts and to routinely assess breast density, not only for its role as a risk factor for breast cancer but also for its potential influence on cancer prognosis. 


\section{Introduction}

Mammographic breast density is defined as the relative amount of radiolucent elements (fatty tissue) and radiopaque elements of the breast (fibroglandular tissue). It has become a key element in breast cancer screening because of its dual effect on breast cancer risk: high breast density impairs the detection of abnormalities in the breast, decreasing the sensitivity of mammography [1], and is also an independent risk factor for breast cancer, as most cancers develop in the glandular parenchyma [2]. More recently, breast density has been postulated as a robust candidate for tailoring screening intervals, suggesting that annual screening may be more effective than biennial screening for women at high risk due to dense breasts in combination with other risk factors [3]. However, such an approach has not been implemented in any screening programme, since it requires more individual-level data, among many other unresolved issues and challenges [4].

Variations in breast density during a woman's lifetime may be influenced by several internal and external factors related to the hormonal environment. Breast density is inversely associated with age, with premenopausal women younger than 50 years being more likely to have dense breasts $[5,6]$. The use of hormone replacement therapy slows the age-related trend to fatty tissue, especially for those women taking a combination of oestrogen and progestin components [7]. In addition, some studies have reported that tumours developing in dense breasts are more likely to express hormone receptors such as oestrogen receptor (ER) and progesterone receptor (PR) $[8,9]$, suggesting a positive association with stromal composition and the oestrogenic microenvironment.

However, whereas increased breast density is a wellrecognised risk factor for breast cancer, the relationship between breast density and breast cancer prognosis is still controversial. Some studies have reported an increased risk of death for women with dense breasts $[10,11]$, while others have found an inverse association [12] or no relationship [13]. In addition, only few studies have been restricted to the context of mammography screening [14-16], also with contradictory results. Because this population has particular characteristics (e.g., average-risk women, women over 45/50 years, mostly postmenopausal), performing studies focused on this population may provide useful information to better understand the relationship between breast density and cancer prognosis and to eventually provide individually tailored screening strategies.

Our aim was to assess the role of breast density on mortality and recurrences, taking into account patient and tumour characteristics and the treatments received among women attending population-based screening programmes.

\section{Materials and Methods}

2.1. Setting and Study Population. This study was carried out among a retrospective cohort of 1,086 women with breast cancer, aged between 50 and 69 years, who underwent breast cancer screening in two Spanish regions (Catalonia and the
Canary Islands; CAMISS retrospective cohort). All of them were diagnosed with breast cancer between 2000 and 2009 and were followed up until June 2014. The study included asymptomatic women with cancers detected in routine screening mammograms and symptomatic women with cancers detected between two screening mammograms (interval cancers)

Mammography screening in Spain follows the recommendations of the European Guidelines for quality assurance in breast cancer screening and diagnosis [17], offering all women aged 50 to 69 years free biennial screening. Two mammographic projections (mediolateral oblique and craniocaudal views) are made, using the BI-RADS (Breast Imaging Reporting and Data System) classification for mammogram reading [18].

As breast density is not routinely recorded by all participating screening programmes, we determined breast density for a subsample of cases. Sample size was calculated to estimate a hazard ratio of 2.5 [15], with a mortality rate of $14.5 \%$ (from the whole CAMISS cohort). With 5\% significance level and $80 \%$ power, 55 subjects were needed in the high-density group. The subsample included all interval cancers with available screening and diagnostic mammograms and a random sample of screen-detected cancers, matched by screening programme and year of cancer diagnosis. After the breast density assessment, this resulted in 375 invasive breast cancers, 79 of them assigned to the highdensity group, thus assuring enough sample size for the analysis.

The study protocol was approved by the Ethics Committee of Parc de Salut Mar, Barcelona (CEIC Parc de Salut Mar). Specific patient consent was not required.

2.2. Breast Density Assessment. For the purpose of this study, breast density was retrospectively evaluated by three experienced radiologists who followed a consensus-based protocol, as detailed elsewhere [19]. In brief, each radiologist determined the breast density of the cancer-free breast at the moment of diagnosis using Boyd's scale, a semiquantitative score of six categories using percentages of density: A: 0\%; B: 1-10\%; C: 10-25\%; D: 25-50\%; E: 50-75\%; F: 75-100\% [20]. For statistical purposes, breast density was collapsed into low ( $\leq 25 \%$ density), intermediate (25-50\% density), and high density ( $\geq 50 \%$ density).

2.3. Study Variables. Patient information, including age at diagnosis, menopausal status, hormone replacement therapy (ever/never), and first-degree family history of breast cancer, was obtained from the databases of the screening programmes. To obtain information on the burden of disease at diagnosis, we manually reviewed clinical records to identify the presence of comorbidities and construct the Charlson comorbidity index (CCI) [21]. The CCI was stratified into three categories: $\mathrm{CCI}=0, \mathrm{CCI}=1$, and $\mathrm{CCI} \geq 2$.

Information on mode of detection was obtained from the screening programme databases and by merging data with population-based cancer registries, the hospital minimum basic dataset, and hospital-based cancer registries. We 
differentiated between breast cancers detected by routine screening mammograms (i.e., screen-detected cancers) and cancers detected between 2 screening mammograms, or within 24 months for women who reached the upper age limit (i.e., interval cancers). Further details on the identification of interval cancers are explained elsewhere [19]. Tumour-related information, including tumour size, lymph node involvement, focality, histological type, histological grade, and biomarker expression, was retrieved from the cancer registries, hospital-based registries, and clinical records. Biomarker expression included information on ER, PR, human epidermal growth factor receptor 2 (Her2), p53, and Ki67 status. The positivity criteria for biomarker expression followed international recommendations and their updates throughout the study period [22,23]. Tumours were classified into the following four phenotypes based on the expression of ER, PR, and Her2: (1) luminal A: ER+/Her2or $\mathrm{PR}+/ \mathrm{Her} 2-$; (2) luminal $\mathrm{B}$ : $\mathrm{ER}+/ \mathrm{Her} 2+$ or $\mathrm{PR}+/ \mathrm{Her} 2+$; (3) Her2: ER-/PR-/Her2+; and (4) triple-negative: ER-, PR-, Her2- [24].

From the review of the clinical records, we obtained information on the treatments received. We considered two types of surgery: radical (including all the mastectomies performed, whether radical or simple) and conservative. Information on breast surgery and axillary lymph node dissection (ALND) treatments was collapsed into a single explicative variable. Information on adjuvant treatment was categorised as follows: chemotherapy, radiotherapy, and hormonal therapy; radiotherapy and hormonal therapy; and other treatments.

2.4. Follow-Up Information. Information on recurrences (including locoregional and distant recurrences), second breast neoplasms, and vital status at the end of follow-up (alive or dead) was obtained from the cancer registries and clinical records. Locoregional recurrence was defined as disease recurrence within the ipsilateral breast or chest wall, in the ipsilateral axillary nodes, internal mammary nodes, or supraclavicular nodes. Distant recurrence was defined as disease recurrence in sites other than the breast or regional lymph nodes (bone, skin, or visceral metastasis). A second neoplasm was considered as a second primary carcinoma developing in the ipsilateral or contralateral breast.

Overall survival was computed from the date of breast cancer diagnosis to death from any cause. Patients were censored at the date of their last hospital visit. Recurrencefree survival was computed from the date of breast cancer diagnosis to the first locoregional or distant recurrence, whichever occurred first. Women lost to follow-up or those who died were censored either at the last visit or at death. The median follow-up period was 8.7 years (interquartile range (IQR): 7.2-10.6).

2.5. Statistical Analyses. Descriptive analyses of patient and tumour characteristics and the treatments received according to breast density categories were explored using contingency tables.
Survival curves for overall mortality and for recurrences were generated by using the Kaplan-Meier method and were compared by the log-rank test. Recurrence-free survival and overall survival were plotted by breast density categories. 5year and 10-year survival rates and their $95 \%$ confidence intervals $(95 \% \mathrm{CI})$ were computed.

We fitted two multivariate Cox proportional hazards regression models to estimate the hazard ratios (HR) and their 95\% CI for death and recurrences using a stepwise backward variable selection approach. The initial model included all predictors. In the final models, we forced to include age, screening programme, and CCI as adjusting variables, although they were not statistically significant. The proportional hazards assumption was ascertained by assessment of log-log survival plots. To test the statistical significance of breast density variable as a whole, we performed a Wald test in both models.

All statistical tests were two-sided. $p$ values $<0.05$ were considered statistically significant. Analyses were performed using the statistical software IBM SPSS Statistics version 23.0 (Armonk, NY, USA) and R statistical software version 3.3.2 (http://www.r-project.org).

\section{Results}

A total of 375 invasive breast cancers were included in this study, most of them detected among women with lowdensity breasts $(51.2 \%, 27.7 \%$, and $21.1 \%$ of tumours detected in women with low-, intermediate-, and high-density breasts).

Patient characteristics by breast density categories are summarized in Table 1. Percentages of women with low breast density were highest among older and postmenopausal women. No differences were observed between a family history of breast cancer, the use of hormone replacement therapy or comorbidities, and breast density categories.

Tumour characteristics according to breast density categories are shown in Table 2. Screen-detected cancers were more common among women with low-density breasts, whereas interval cancers were more frequent in intermediate- and high-density breasts. Tumours detected in low-density breasts showed a trend to be smaller, nodenegative, unifocal, and triple-negative. No differences were observed among the treatments received, although the percentage of radical surgery tended to be higher among women with dense breasts.

Kaplan-Meier curves revealed poorer overall survival $(p=0.010)$ and poorer relapse-free survival $(p=0.032)$ among women with high-density breasts (Figure 1). 5-year overall survival rate for women with low breast density was 0.97 (95\% CI: 0.94-1.00), whereas figures for women with high breast density were 0.83 (95\% CI: $0.72-0.96)$. The same pattern was observed at 10 years of follow-up. Recurrencefree survival rate at 5 years was $0.97(0.94-1.00)$ for women with low breast density and $0.81(0.69-0.95)$ for women with high breast density (Table 3).

Adjusted Cox proportional hazards regression models revealed that breast density was statistically significant for 
TABLe 1: Patient-related characteristics by breast density categories.

\begin{tabular}{|c|c|c|c|c|}
\hline & $\begin{array}{c}\text { Total } n=375 \\
(\%)\end{array}$ & $\begin{array}{l}\text { Low breast density }(<25 \%) \\
n=192(\%)\end{array}$ & $\begin{array}{l}\text { Intermediate breast density }(25-50 \%) \\
n=104(\%)\end{array}$ & $\begin{array}{c}\text { High breast density }(>50 \%) \\
n=79(\%)\end{array}$ \\
\hline \multicolumn{5}{|c|}{ Age groups (years) } \\
\hline $50-54$ & $106(28.3)$ & $34(17.7)$ & $33(31.7)^{\mathrm{a}}$ & $39(49.4)$ \\
\hline $55-59$ & $102(27.2)$ & $50(26)$ & $33(31.7)$ & $19(24.1)$ \\
\hline $60-64$ & $103(27.5)$ & $64(33.3)$ & $24(23.1)$ & $15(19)$ \\
\hline $65-70$ & $64(17.1)$ & $44(22.9)$ & $14(13.5)$ & $6(7.6)$ \\
\hline \multicolumn{5}{|l|}{ Menopausal status } \\
\hline Premenopause & $31(13.3)$ & $5(4.7)$ & $9(12.3)$ & $17(32.1)$ \\
\hline Menopause & $202(86.7)$ & $102(95.3)$ & $64(87.7)$ & $36(67.9)$ \\
\hline \multicolumn{5}{|c|}{ Hormone replacement therapy } \\
\hline No & $192(85)$ & $86(84.3)$ & $60(84.5)$ & $46(86.8)$ \\
\hline Yes & $34(15)$ & $16(15.7)$ & $11(15.5)$ & $7(13.2)$ \\
\hline \multicolumn{5}{|c|}{ Family history of breast cancer } \\
\hline No & $200(86.6)$ & $90(85.7)$ & $63(86.3)$ & $47(88.7)$ \\
\hline Yes & $31(13.4)$ & $15(14.3)$ & $10(13.7)$ & $6(11.3)$ \\
\hline \multicolumn{5}{|c|}{ Charlson comorbidity index } \\
\hline 0 & $285(76)$ & $143(74.5)$ & $79(76)$ & $63(79.7)$ \\
\hline 1 & $53(14.1)$ & $31(16.1)$ & $13(12.5)$ & $9(11.4)$ \\
\hline 2 & $37(9.9)$ & $18(9.4)$ & $12(11.5)$ & $7(8.9)$ \\
\hline
\end{tabular}

predicting mortality (Wald test $p$ value $=0.050)$ but not for predicting recurrences (Wald test $p$ value $=0.499$ ). Women with intermediate- and high-density breasts had a higher risk of death than women with low-density breasts, reaching statistical significance for intermediate densities (Table 4) $(\mathrm{aHR}=2.19 \quad[95 \% \quad \mathrm{CI}: 1.16-4.13], \quad \mathrm{aHR}=1.44 \quad[95 \% \mathrm{CI}:$ $0.67-3.10]$, for intermediate and high densities, respectively). Tumours arising as interval cancers $(\mathrm{aHR}=1.96$ [95\% CI: 1.09-3.52] and node-positive tumours were also associated with a higher risk of death $(\mathrm{aHR}=2.73$ [95\% CI: 1.55-4.81]) in the adjusted model (data not shown).

Breast density showed no association with the risk of recurrences $(\mathrm{aHR}=1.43$ [95\% CI: 0.71-2.89]; $\mathrm{aHR}=1.47$ [95\% CI: 0.71-3.08], for intermediate and high densities, respectively) (Table 5). Node-positive tumours showed an increased risk of recurrences in the adjusted analysis $(\mathrm{aHR}=3.96$ [95\% CI: 2.12-7.39]) (data not shown).

\section{Discussion}

The results of the current study suggest that higher breast density is associated with a greater risk of death in women participating in breast cancer screening, while breast density showed no association with the risk of recurrences.

The positive association between dense tissue and risk of death is consistent with some $[14,15]$, but not all $[16,25]$, prior studies conducted among screened women. Based on the data of Swedish women, Chiu et al. found that dense tissue increased mortality from breast cancer in addition to increasing breast cancer risk and the likelihood of more aggressive tumours [14]. Based on Danish data, Olsen et al. also found a positive association between dense tissue and death, although they reported lower case fatality among tumours developing in dense breasts [15]. By contrast, a study carried out in the UK [25] reported no relationship between breast density and survival. In that study, however, the screening interval was 3 years, and the survival analyses were not adjusted. A recent study carried out among the Dutch population also reported no relationship between breast density and survival [16]. In this study, as pointed out by the authors, the lack of tumour-related information may confound the results shown. In addition, the definition for high density includes $\geq 25 \%$ of dense tissue, differing from most of the published studies. Other works conducted in nonscreening populations have also found contradictory results, some of them reporting positive associations between breast density and mortality $[10,11]$ and others finding no association [13] or even a negative association [12].

Some authors have hypothesized that the association between higher density and worse survival would be explained by the diagnosis delay due to the masking effect. In that sense, we do observe a higher percentage of larger, node-positive, and interval cancers among women with high-density breasts in the descriptive data. In the adjusted analyses, lymph node involvement and detection as an interval cancer were also associated with mortality, along with intermediate breast densities so that the current results would support this hypothesis, since breast density as well as other factors related to diagnostic delay remained associated with the risk of death. These findings reinforce the need to improve screening sensitivity among women with dense breasts, which is currently been proposed by means of shifting the conventional one-size-fits-all screening approach towards more personalized screening strategies based on the individual risk of breast cancer.

Other authors have postulated that the relationship between breast density and survival may be explained by the tumour characteristics of cancers arising in epithelial tissue. It has been suggested an increased proliferation and growth factors in dense tissue $[26,27]$ that may be involved in pathways that lead to more aggressive tumours. Nevertheless, the evidence supporting this hypothesis is not conclusive and 
TABLE 2: Tumour characteristics by breast density categories.

\begin{tabular}{|c|c|c|c|c|}
\hline & $\begin{array}{c}\text { Total } \\
n=375(\%)\end{array}$ & $\begin{array}{l}\text { Low breast density } \\
(<25 \%) n=192(\%)\end{array}$ & $\begin{array}{l}\text { Intermediate breast density } \\
\quad(25-50 \%) n=104(\%)\end{array}$ & $\begin{array}{l}\text { High breast density } \\
(>50 \%) n=79(\%)\end{array}$ \\
\hline \multicolumn{5}{|l|}{ Mode of detection } \\
\hline Screen-detected cancers & $195(52)$ & $113(58.9)$ & $45(43.3)$ & $37(46.8)$ \\
\hline Interval cancer & $180(48)$ & $79(41.1)$ & $59(56.7)$ & $42(53.2)$ \\
\hline \multicolumn{5}{|l|}{ Tumour size } \\
\hline$<20 \mathrm{~mm}$ & $199(61.8)$ & $108(56.3)$ & $53(51)$ & $38(48.1)$ \\
\hline$\geq 20 \mathrm{~mm}$ & $123(38.2)$ & $58(30.2)$ & $33(31.7)$ & $32(40.5)$ \\
\hline \multicolumn{5}{|l|}{ Lymph node involvement } \\
\hline Negative & $218(66.1)$ & $117(60.9)$ & $60(57.7)$ & $41(51.9)$ \\
\hline Positive & $112(33.9)$ & $51(26.6)$ & $30(28.8)$ & $31(39.2)$ \\
\hline \multicolumn{5}{|l|}{ Focality } \\
\hline Unifocal & $301(84.6)$ & $155(87.1)$ & $80(80)$ & $66(84.6)$ \\
\hline Multifocal and/or multicentric & $55(15.4)$ & $23(12.9)$ & $20(20)$ & $12(15.4)$ \\
\hline \multicolumn{5}{|l|}{ Histological type } \\
\hline Ductal & $303(80.8)$ & $152(79.2)$ & $86(82.7)$ & $65(82.3)$ \\
\hline Lobular & $39(10.4)$ & $22(11.5)$ & $8(7.7)$ & $9(11.4)$ \\
\hline Others & $33(8.8)$ & $18(9.4)$ & $10(9.6)$ & $5(6.3)$ \\
\hline \multicolumn{5}{|l|}{ Histological grade } \\
\hline I & $83(23.9)$ & $44(22.9)$ & $18(17.3)$ & $21(26.6)$ \\
\hline II & $134(38.5)$ & $64(33.3)$ & $40(38.5)$ & $30(38)$ \\
\hline III & $116(33.3)$ & $60(31.3)$ & $35(33.7)$ & $21(26.6)$ \\
\hline NA & $15(4.3)$ & $9(4.7)$ & $3(2.9)$ & $3(3.8)$ \\
\hline \multicolumn{5}{|l|}{ Oestrogen receptor } \\
\hline Negative & $88(23.5)$ & $46(24)$ & $28(26.9)$ & $14(17.7)$ \\
\hline Positive & $287(76.5)$ & $146(76)$ & $76(73.1)$ & $65(82.3)$ \\
\hline \multicolumn{5}{|l|}{ Progesterone receptor } \\
\hline Negative & $150(40.1)$ & $70(36.5)$ & $45(43.3)$ & $35(44.3)$ \\
\hline Positive & $224(59.9)$ & $121(63)$ & $59(56.7)$ & $44(55.7)$ \\
\hline \multicolumn{5}{|l|}{ HER2 } \\
\hline Negative & $261(79.1)$ & $133(69.3)$ & $70(67.3)$ & $58(73.4)$ \\
\hline Positive & $69(20.9)$ & $35(18.2)$ & $21(20.2)$ & $13(16.5)$ \\
\hline \multicolumn{5}{|l|}{ Ki67 } \\
\hline Negative & $114(60.3)$ & $62(32.3)$ & $29(27.9)$ & $23(29.1)$ \\
\hline Positive & $75(39.7)$ & $49(25.5)$ & $15(14.4)$ & $11(13.9)$ \\
\hline \multicolumn{5}{|l|}{ Tumour phenotype } \\
\hline Luminal A & $155(48)$ & $79(41.1)$ & $38(36.5)$ & $38(48.1)$ \\
\hline Luminal B & $93(28.8)$ & $46(24)$ & $26(25)$ & $21(26.6)$ \\
\hline HER2 & $31(9.6)$ & $11(5.7)$ & $12(11.5)$ & $8(10.1)$ \\
\hline Triple-negative & $44(13.6)$ & $29(15.1)$ & $11(10.6)$ & $4(5.1)$ \\
\hline \multicolumn{5}{|l|}{ Treatment } \\
\hline $\begin{array}{l}\text { Conservative surgery only or } \\
\text { with sentinel lymph node biopsy }\end{array}$ & $97(26.4)$ & $50(26.4)$ & $28(28)$ & $19(24.1)$ \\
\hline $\begin{array}{l}\text { Conservative surgery with } \\
\text { axillary lymph node dissection }\end{array}$ & $182(49.5)$ & $100(52.9)$ & $44(44)$ & $38(48.1)$ \\
\hline $\begin{array}{l}\text { Radical surgery with or without } \\
\text { lymphadenectomy }\end{array}$ & $79(21.5)$ & $35(18.5)$ & $24(24)$ & $20(25.3)$ \\
\hline $\begin{array}{l}\text { No surgery and/or adjuvant } \\
\text { treatment }\end{array}$ & $10(2.7)$ & $4(2.1)$ & $4(4)$ & $2(2.5)$ \\
\hline \multicolumn{5}{|l|}{ Adjuvant treatment after surgery } \\
\hline $\begin{array}{l}\text { Chemotherapy, radiotherapy, } \\
\text { and hormonal therapy }\end{array}$ & $122(32.5)$ & $54(28.1)$ & $36(34.6)$ & $32(40.5)$ \\
\hline $\begin{array}{l}\text { Radiotherapy and hormonal } \\
\text { therapy }\end{array}$ & $123(32.8)$ & $68(35.4)$ & $35(33.7)$ & $20(25.3)$ \\
\hline Other treatments & $130(34.7)$ & $70(36.5)$ & $33(31.7)$ & $27(34.2)$ \\
\hline
\end{tabular}

seems contradictory to the overrepresentation (although nonsignificant) of triple-negative cancers in low-density breasts, observed in the current descriptive data and in previous works $[19,28]$. Further studies conducted in larger cohorts, with information on breast density, tumour characteristics, and clinical outcomes, are warranted to elucidate the mechanisms through which breast density and prognosis are associated.

Contrasting with prior series, we did not find association between breast density and the risk of recurrences [29-31]. 
TABLE 3: 5-year and 10-year survival rates for overall survival and recurrence-free survival.

\begin{tabular}{lcc}
\hline & 5-year survival rate (95\% CI) & 10-year survival rate (95\% CI) \\
\hline Overall survival & & \\
$\quad$ Low breast density $(<25 \%)$ & $0.97(0.94-1.00)$ & $0.95(0.91-0.99)$ \\
Intermediate breast density $(25-50 \%)$ & $0.89(0.80-0.99)$ & $0.82(0.71-0.96)$ \\
High breast density (>50\%) & $0.83(0.72-0.96)$ & $0.78(0.65-0.93)$ \\
Recurrence-free survival & $0.97(0.94-1.00)$ & $0.92(0.85-0.98)$ \\
Low breast density (<25\%) & $0.93(0.86-1.00)$ & $0.88(0.79-0.99)$ \\
Intermediate breast density (25-50\%) & $0.81(0.69-0.95)$ & $0.78(0.65-0.93)$ \\
High breast density (>50\%) & \\
\hline
\end{tabular}

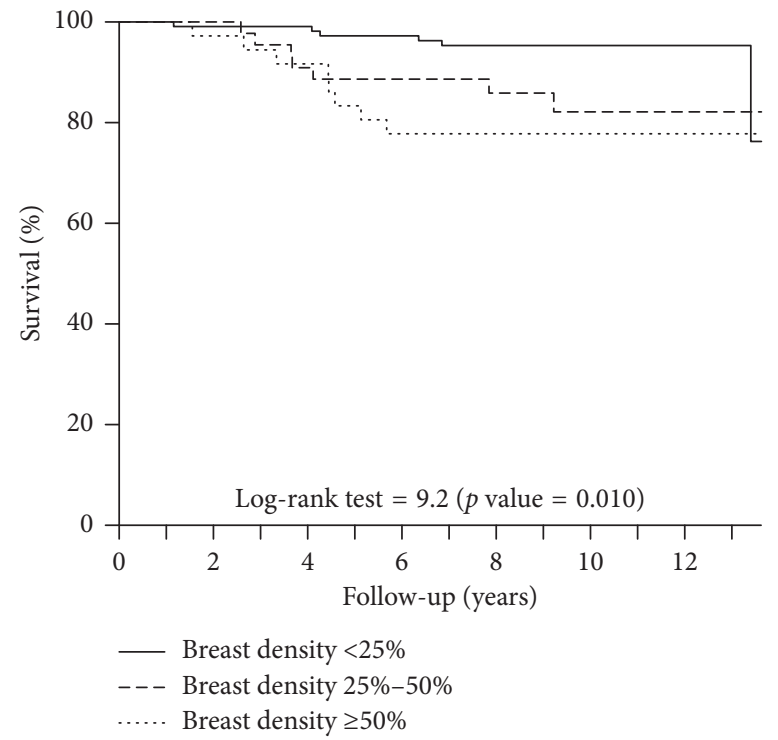

(a)

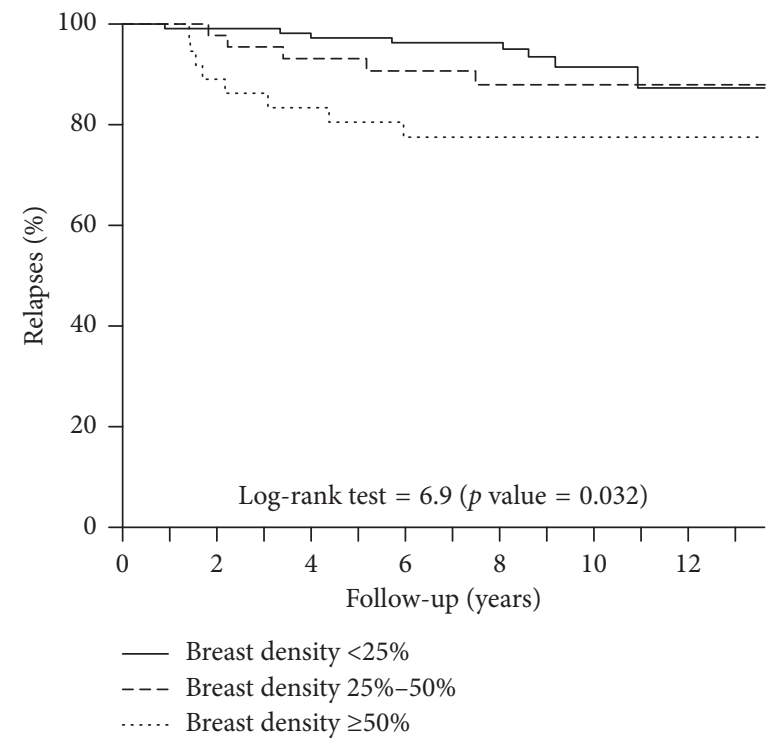

(b)

Figure 1: Survival and recurrence-free survival by breast density. (a) Overall survival; log-rank test $=0.010$. (b) Recurrence-free survival; logrank test $=0.032$.

TABLE 4: Unadjusted and adjusted hazard ratios for death.

\begin{tabular}{|c|c|c|c|}
\hline & Number of deaths & Unadjusted HR & Adjusted HR* \\
\hline \multicolumn{4}{|c|}{ Breast density } \\
\hline$<25 \%$ & 20 & & $\operatorname{Re}$ \\
\hline $25-50 \%$ & 24 & 248 & $2.19(1.16-4$ \\
\hline$>50 \%$ & 15 & $1.89(0.97-3.7)$ & $1.44(0.67-3.10)$ \\
\hline
\end{tabular}

*The final model included breast density, mode of detection, lymph node involvement, age, Charlson comorbidity index, and screening programme. HR: hazard ratio; aHR: adjusted hazard ratio.

TABLE 5: Unadjusted and adjusted hazard ratios for recurrences.

\begin{tabular}{lccc}
\hline & $\begin{array}{c}\text { Number of } \\
\text { recurrences }\end{array}$ & $\begin{array}{c}\text { Unadjusted } \\
\text { HR }\end{array}$ & Adjusted HR* \\
\hline $\begin{array}{l}\text { Breast density } \\
\leq 25 \%\end{array}$ & 19 & Ref. & Ref. \\
$25-50 \%$ & 16 & 1.72 & 1.43 \\
& & $(0.89-3.35)$ & $(0.71-2.89)$ \\
$\geq 50 \%$ & 17 & $2.34(1.21-4.5)$ & 1.47 \\
& & & $(0.71-3.08)$ \\
\hline
\end{tabular}

*Adjusted by breast density, progesterone receptor, lymph node involvement, age, Charlson comorbidity index, and screening programme. HR: hazard ratio; aHR: adjusted hazard ratio.
Those studies reported an increased risk of locoregional recurrences, but not for distant metastasis or death. Unfortunately, our study sample was not large enough to replicate the analysis for different types of recurrence. Besides, the populations considered in these works differed from ours, since they included study periods prior to ours, which could involve different treatment schemes. In addition, the study by Park et al. [30] only included patients undergoing breast-conserving surgery and radiotherapy, whereas we included both patients receiving and not receiving radiotherapy, which is strongly related to the risk of recurrences [29]. Our adjusted analyses revealed that the only factor associated with recurrences was the lymph node involvement at diagnosis.

Our study is limited by the relatively small number of events in some categories, which prevented us from including all breast density categories of Boyd's scale in the adjusted model. Nevertheless, most studies assessing the effect of breast density on mortality outcomes collapse breast density data into two or three categories, making our data more comparable with those of previous works. Second, we were not able to explore breast-specific cancer mortality. Previous studies exploring both breast-specific cancer 
mortality and mortality from other causes found that the latter was not associated with breast density [11]. Therefore, the impact of analysing all causes of death together may lead to underestimation of the effect of breast density on mortality. Finally, we used a qualitative classification for breast density assessment, which is known to have moderate interobserver concordance [32]. However, to minimise misclassification, breast density assessment was centralised and performed by a panel of experienced radiologists, specially trained for the study [19].

The current study is strengthened by the homogeneity of the study population included. Restricting the study to screening participants allowed us to explore the effect of breast density on a relatively homogeneous group of patients in terms of age range and tumour stage. Thus, the conclusions drawn from the current work are robust and informative within the framework of population-based screening and are of interest for tailored screening strategies. In addition, the availability of data on comorbidities, patient and tumour characteristics, and the treatments received allowed us to control for important prognostic factors and to explore-for the first time among women participating in breast cancer screening-the effect of breast density on mortality considering both patient and tumour characteristics and treatments received.

\section{Conclusion}

In conclusion, our findings reveal that increased breast density was associated with worse survival outcomes among women participating in breast cancer screening. This association seems to be mainly explained as a result of the masking effect of dense tissue, although an underlying biological mechanism in the stroma composition may also play a role. These findings reinforce the need to improve screening sensitivity among women with dense breasts by means of more personalized screening approaches as well as the importance to routinely assess and record information on breast density during the screening process, both because of its utility as a predictive factor for breast cancer and because of its role in breast cancer prognosis.

\section{Abbreviations}

ALND: Axillary lymph node dissection

BI-RADS: Breast Imaging Reporting and Data System

CCI: $\quad$ Charlson comorbidity index

ER: Oestrogen receptor

Her2: Human epidermal growth factor receptor 2

HR: Hazard ratios

IQR: Interquartile range

PR: $\quad$ Progesterone receptor

TNM: Tumour-node-metastasis.

\section{Data Availability}

The retrospective observational data used to support the findings of this study are available from the corresponding author on reasonable request.

\section{Conflicts of Interest}

The authors declare that they have no conflicts of interest.

\section{Acknowledgments}

The authors acknowledge the contribution of Francesc Macià, Imma Collet, and the Hospital del Mar Tumour Registry (Barcelona) in providing tumour-related data. The authors also would like to acknowledge the dedication and support of the entire CAMISS Study Group (in alphabetical order): IMIM (Hospital del Mar Medical Research Institute), Barcelona: Xavier Castells, Mercè Comas, Laia Domingo, Francesc Macià, Marta Roman, Anabel Romero, Maria Sala; Canary Islands Health Service: Teresa Barata, Isabel Diez de la Lastra, Mariola de la Vega; Corporació Sanitaria Parc Taulí, Sabadell: Marisa Baré, Núria Torà; Hospital Santa Caterina, Girona: Joana Ferrer; Epidemiology Unit and Girona Cancer Registry: Maria Carmen Carmona-Garcia; Hospital GaldakaoUsansolo, Vizcaya: Susana García, Maximina Martín, Miren Orive, Maria Amparo Valverde; Canary Islands Foundation for Health Research: Jeanette Pérez, Amado Rivero, Cristina Valcárcel; Hospital Costa del Sol, University of Málaga: María del Carmen Padilla, Maximino Redondo, Teresa Téllez, Irene Zarcos; Hospital Universitario Donostia/Biodonostia: Cristina Churruca, Amaia Perales, Javier Recio, Irune Ruiz, Cristina Sarasqueta, Jose María Urraca; Instituto Oncológico de Guipúzcoa-Onkologikoa: Maria Jesús Michelena; Hospital Universitario Basurto: Julio Moreno; Hospital Universitario Cruces: Gaizka Mallabiabarrena, Patricia Cobos, Borja Otero; Hospital Universitario Txagorritxu: Javier Gorostiaga, Itsaso Troya. This study was supported by grants from Instituto de Salud Carlos III FEDER (grant numbers: PS09/01153, PI12/00387, PI11/01296, PI15/ 00098, and PI16/00244) and by the Research Network on Health Services in Chronic Diseases (Instituto de Salud Carlos III) (REDISSEC: RD12/0001/0015, RD12/0001/ 0007, and RD16/0001/0013).

\section{References}

[1] D. van der Waal, T. M. Ripping, A. L. M. Verbeek, and M. J. M. Broeders, "Breast cancer screening effect across breast density strata: a case-control study," International Journal of Cancer, vol. 140, no. 1, pp. 41-49, 2017.

[2] N. F. Boyd, H. Guo, L. J. Martin et al., "Mammographic density and the risk and detection of breast cancer," New England Journal of Medicine, vol. 356, no. 3, pp. 227-236, 2007.

[3] A. Trentham-Dietz, K. Kerlikowske, N. K. Stout et al., "Tailoring breast cancer screening intervals by breast density and risk for women aged 50 years or older: collaborative modeling of screening outcomes," Annals of Internal Medicine, vol. 165, no. 10, pp. 700-712, 2016.

[4] L. Rainey, D. van der Waal, A. Jervaeus et al., "Are we ready for the challenge of implementing risk-based breast cancer screening and primary prevention?," The Breast, vol. 39, pp. 24-32, 2018. 
[5] C. M. Checka, J. E. Chun, F. R. Schnabel, J. Lee, and H. Toth, "The relationship of mammographic density and age: implications for breast cancer screening," American Journal of Roentgenology, vol. 198, no. 3, pp. W292-W295, 2012.

[6] M. E. Work, L. L. Reimers, A. S. Quante, K. D. Crew, A. Whiffen, and M. B. Terry, "Changes in mammographic density over time in breast cancer cases and women at high risk for breast cancer," International Journal of Cancer, vol. 135, no. 7, pp. 1740-1744, 2014.

[7] F. J. B. van Duijnhoven, P. H. M. Peeters, R. M. L. Warren et al., "Postmenopausal hormone therapy and changes in mammographic density," Journal of Clinical Oncology, vol. 25, no. 11, pp. 1323-1328, 2007.

[8] L. Yaghjyan, G. A. Colditz, L. C. Collins et al., "Mammographic breast density and subsequent risk of breast cancer in postmenopausal women according to tumor characteristics," JNCI Journal of the National Cancer Institute, vol. 103, no. 15, pp. 1179-1189, 2011.

[9] J. Ding, R. Warren, A. Girling, D. Thompson, and D. Easton, "Mammographic density, estrogen receptor status and other breast cancer tumor characteristics," The Breast Journal, vol. 16, no. 3, pp. 279-289, 2010.

[10] A. Olsson, H. Sartor, S. Borgquist, S. Zackrisson, and J. Manjer, "Breast density and mode of detection in relation to breast cancer specific survival: a cohort study," BMC Cancer, vol. 14, p. 229, 2014.

[11] G. Maskarinec, I. S. Pagano, M. A. Little, S. M. Conroy, S.-Y. Park, and L. N. Kolonel, "Mammographic density as a predictor of breast cancer survival: the multiethnic cohort," Breast Cancer Research, vol. 15, no. 1, p. R7, 2013.

[12] A. Masarwah, P. Auvinen, M. Sudah et al., "Very low mammographic breast density predicts poorer outcome in patients with invasive breast cancer," European Radiology, vol. 25, no. 7, pp. 1875-1882, 2015.

[13] G. L. Gierach, L. Ichikawa, K. Kerlikowske et al., "Relationship between mammographic density and breast cancer death in the Breast Cancer Surveillance Consortium," JNCI: Journal of the National Cancer Institute, vol. 104, no. 16, pp. 1218-1227, 2012.

[14] S. Y. H. Chiu, S. Duffy, A. M. F. Yen, L. Tabar, R. A. Smith, and H. H. Chen, "Effect of baseline breast density on breast cancer incidence, stage, mortality, and screening parameters: 25-year follow-up of a Swedish mammographic screening," Cancer Epidemiology Biomarkers \& Prevention, vol. 19, no. 5, pp. 1219-1228, 2010.

[15] A. H. Olsen, K. Bihrmann, M.-B. Jensen, I. Vejborg, and E. Lynge, "Breast density and outcome of mammography screening: a cohort study," British Journal of Cancer, vol. 100, no. 7, pp. 1205-1208, 2009.

[16] D. van der Waal, A. L. M. Verbeek, and M. J. M. Broeders, "Breast density and breast cancer-specific survival by detection mode," BMC Cancer, vol. 18, 2018.

[17] European Commission and Directorate-General for Health and Consumer Protection, European Guidelines for Quality Assurance in Breast Cancer Screening and Diagnosis, N. Perry and E. Puthaar, Eds., Office for Official Publications of the European Communities, Brussels, Belgium, 2006.

[18] E. A. Sickles and C. J. B. L. D’Orsi, "ACR BI-RADS ${ }^{\circledR}$ mammography," in ACR BI-RADS ${ }^{\circledR}$ Atlas, Breast Imaging Reporting and Data System, American College of Radiology, Reston, VA, USA, 2013.

[19] L. Domingo, D. Salas, R. Zubizarreta et al., "Tumor phenotype and breast density in distinct categories of interval cancer: results of population-based mammography screening in Spain," Breast Cancer Research, vol. 16, no. 1, p. R3, 2014.

[20] N. F. Boyd, J. W. Byng, R. A. Jong et al., "Quantitative classification of mammographic densities and breast cancer risk: results from the Canadian National Breast Screening Study," JNCI Journal of the National Cancer Institute, vol. 87, no. 9, pp. 670-675, 1995.

[21] M. E. Charlson, P. Pompei, K. L. Ales, and C. R. MacKenzie, "A new method of classifying prognostic comorbidity in longitudinal studies: development and validation," Journal of Chronic Diseases, vol. 40, no. 5, pp. 373-383, 1987.

[22] A. C. Wolff, M. E. H. Hammond, J. N. Schwartz et al., "American Society of Clinical Oncology/College of American Pathologists guideline recommendations for human epidermal growth factor receptor 2 testing in breast cancer," Journal of Clinical Oncology, vol. 25, no. 1, pp. 118-145, 2006.

[23] M. E. H. Hammond, D. F. Hayes, M. Dowsett et al., "American Society of Clinical Oncology/College of American Pathologists guideline recommendations for immunohistochemical testing of estrogen and progesterone receptors in breast cancer (unabridged version)," Archives of Pathology \& Laboratory Medicine, vol. 134, pp. e48-e72, 2010.

[24] A. Goldhirsch, W. C. Wood, A. S. Coates et al., "Strategies for subtypes-dealing with the diversity of breast cancer: highlights of the St Gallen international expert consensus on the primary therapy of early breast cancer 2011," Annals of Oncology, vol. 22, no. 8, pp. 1736-1747, 2011.

[25] G. J. R. Porter, A. J. Evans, E. J. Cornford et al., "Influence of mammographic parenchymal pattern in screening-detected and interval invasive breast cancers on pathologic features, mammographic features, and patient survival," American Journal of Roentgenology, vol. 188, no. 3, pp. 676-683, 2007.

[26] N. F. Boyd, L. J. Martin, M. J. Yaffe, and S. Minkin, "Mammographic density and breast cancer risk: current understanding and future prospects," Breast Cancer Research, vol. 13, p. 223, 2011.

[27] M. Ellingjord-Dale, E. Lee, E. Couto et al., "Polymorphisms in hormone metabolism and growth factor genes and mammographic density in Norwegian postmenopausal hormone therapy users and non-users," Breast Cancer Research, vol. 14, no. 5, p. R135, 2012.

[28] V. A. Kirsh, A. M. Chiarelli, S. A. Edwards et al., "Tumor characteristics associated with mammographic detection of breast cancer in the Ontario breast screening program," JNCI Journal of the National Cancer Institute, vol. 103, no. 12, pp. 942-950, 2011.

[29] T. Cil, E. Fishell, W. Hanna et al., "Mammographic density and the risk of breast cancer recurrence after breast-conserving surgery," Cancer, vol. 115, no. 24, pp. 5780-5787, 2009.

[30] C. C. Park, J. Rembert, K. Chew, D. Moore, and K. Kerlikowske, "High mammographic breast density is independent predictor of local but not distant recurrence after lumpectomy and radiotherapy for invasive breast cancer," International Journal of Radiation Oncology · biology · physics, vol. 73, no. 1, pp. 75-79, 2009.

[31] L. Eriksson, K. Czene, L. U. Rosenberg, S. Törnberg, K. Humphreys, and P. Hall, "Possible influence of mammographic density on local and locoregional recurrence of breast cancer," Breast Cancer Research, vol. 15, no. 4, 2013.

[32] R. R. Winkel, M. von Euler-Chelpin, M. Nielsen et al., "Interobserver agreement according to three methods of evaluating mammographic density and parenchymal pattern in a case control study: impact on relative risk of breast cancer," BMC Cancer, vol. 15, p. 274, 2015. 


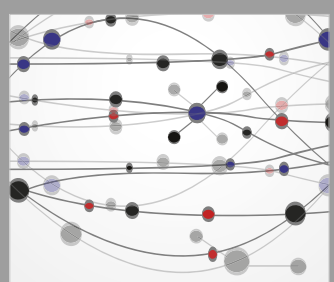

The Scientific World Journal
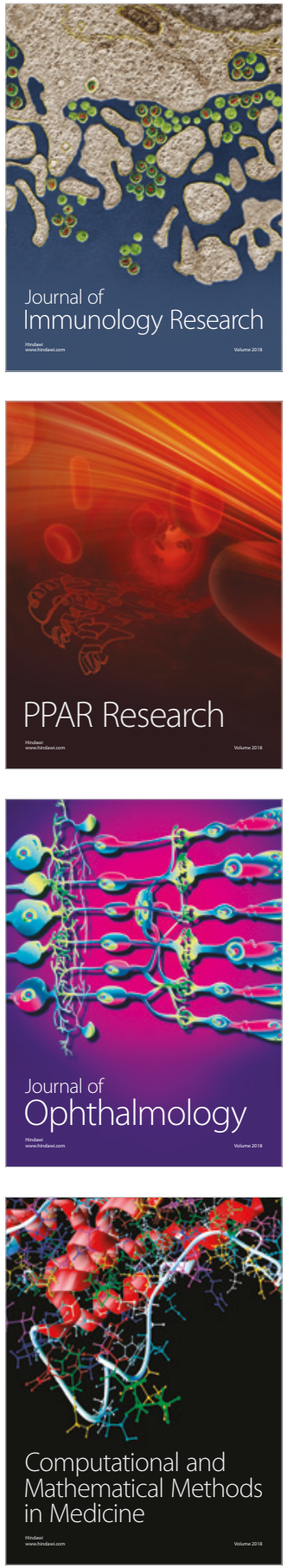

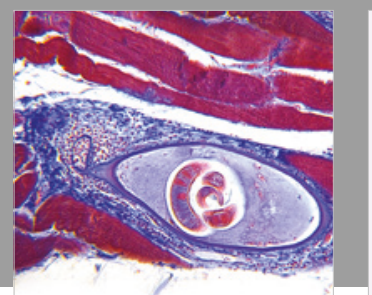

Gastroenterology Research and Practice

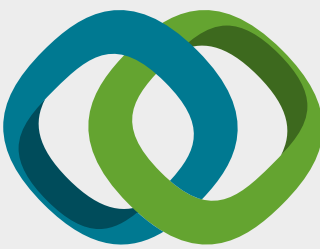

\section{Hindawi}

Submit your manuscripts at

www.hindawi.com
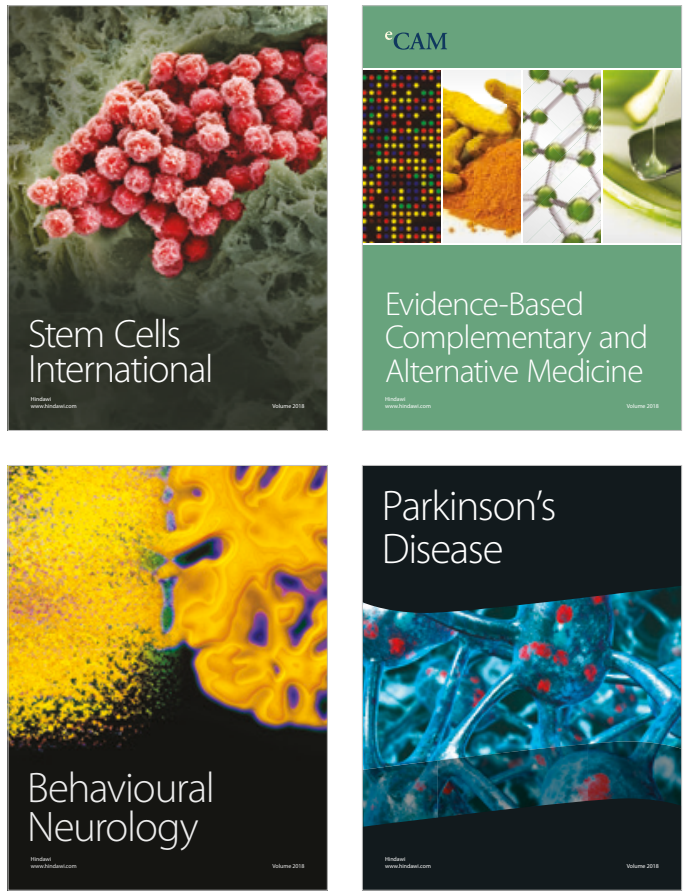

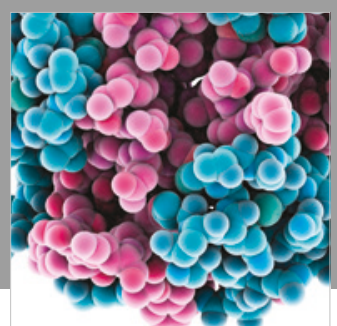

ournal of

Diabetes Research

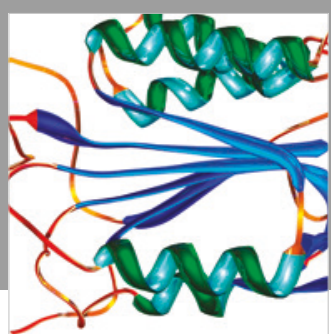

Disease Markers
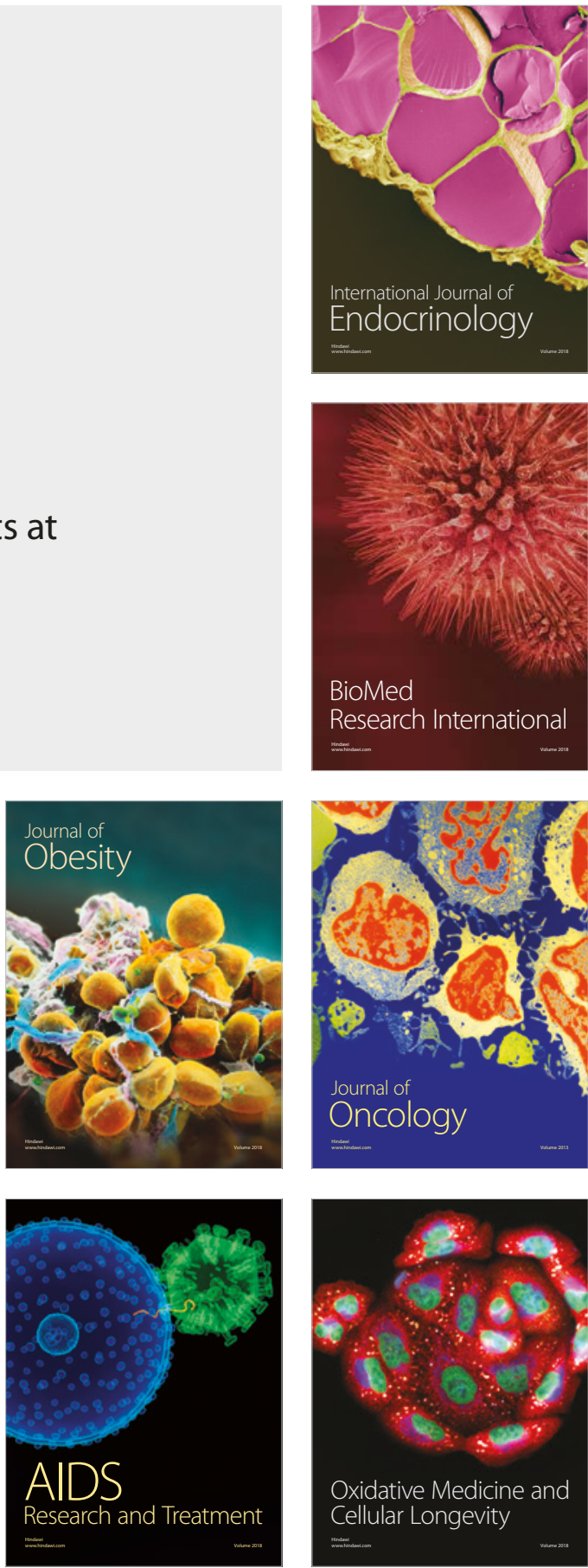